\title{
DESARROLLO EMPRESARIAL Y HUMANO DE ASOCIADOS DE LAS COOPERATIVAS COOPROVASEB R.L Y COOPRAHORT R.L. MUNICIPIOS DE SÉBACO Y DARÍO, MATAGALPA, 2012
}

\section{ENTREPRENEURSHIP AND HUMAN DEVELOPMENT FROM ASSOCIATES OF THE COOPERATIVES COOPROVA- SEB AND COOPRAHORT RL IN THE MUNICIPALITIES OF SEBACO AND DARIO, MATAGALPA, 2012}

López-Aldana Wendy Celeste

Universidad Nacional Autónoma de Nicaragua, UNAN-Managua. (lopezaldanaw@yahoo.es)

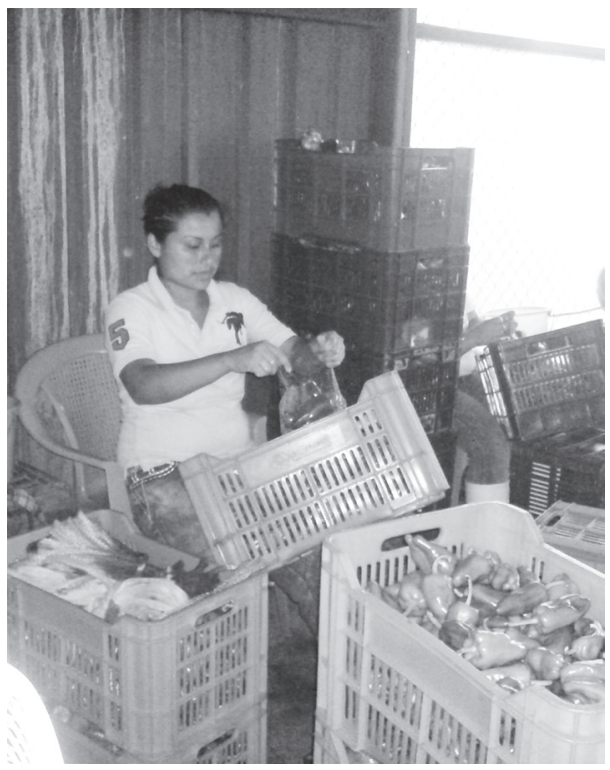

\section{RESUMEN}

El proyecto cadena de valor ejecutado por el Instituto de Desarrollo Rural (IDR) en la zona seca del departamento de Matagalpa (Terrabona, Darío y Sébaco), contempla el fortalecimiento empresarial de las cooperativas productoras de hortalizas de la zona. A solicitud del IDR se realizó un estudio sobre el desarrollo empresarial y humano de las cooperativas Cooprahort, R.L y Cooprovaseb, R.L en el que se determinaron los puntos críticos, así como las ventajas comparativas que presentan cada una de ellas, tomando en cuenta las condiciones de vida de sus asociados. El estudio es de tipo descriptivo, de corte transversal, se utilizó un enfoque mixto, haciendo uso del método teórico mediante fuentes bibliográficas, y el método empírico con la aplicación de encuestas, entrevistas, guía de observación y grupos focales. Mediante el análisis de indicadores como valores y principios cooperativos y toma de decisión, se determinó que los cooperados hacen uso de los deberes y derechos cooperativos; financiamiento, canales de comercialización y control interno, además, con estos indicadores se determinó el nivel de desarrollo empresarial con que cuentan estas organizaciones. Para la determinación del nivel de desarrollo humano se utilizaron indicadores como el ingreso, vivienda, y participación. Se logró determinar que Cooprahort tiene un nivel de desarrollo empresarial opuesto al de Cooprovaseb; la primera cuenta con toda la infraestructura y maquinaria necesaria para llevar a cabo el suministro de la producción a los diferentes clientes, en cambio, la última no dispone de la misma, por lo cual hace que sea totalmente opuesta a Cooprahort. R.L. A pesar de lo anterior, las condiciones de vida de los asociados de ambas cooperativas son similares, aunque el móvil para lograr bienestar no es el mismo. Los asociados de Cooprahort manifesta-

\section{ABSTRACT}

The value chain project implemented by the Rural Development Institute (IDR) in the dry zone of the department of Matagalpa (Terrabona, Dario and Sébaco ) involve the enterprise strengthen of the cooperative of vegetable production in the area At IDR request, a study was conducted on human and enterprise development of the cooperatives Cooprahort, RL and Cooprovaseb, on which the critical points were measured, as well as the comparative advantages of each of them, taking into account the living conditions of its members. The study was cross-sectional, descriptive, using a mixed approach, focusing on the theoretical method by using literature sources and in the empirical method, with the use of surveys, interviews, observation guide and focus groups. Through the analysis of indicators such as cooperative values and principles and decision-making, it was found that the cooperative make use of cooperative rights and obligations, financing, marketing channels and internal control, additionally, with these indicators, it was also determined the level of enterprise development that feature these organizations. In order to determine the level of human development, the following indicators were used: income, housing, and participation. It was possible to determine that Cooprahort have an enterprise development level opposite to Cooprovaseb. Cooprahort features all the infrastructure and equipment necessary to carry out the delivery of production to different clients. On the other hand, Cooprovaseb, does not have it, and therefore makes it totally opposite to Cooprahor, RL. Despite of this, the living conditions of the members of both cooperatives are similar, although the way to achieve welfare is not the same. Cooprahort associates demonstrated that through the cooperative have managed to improve their way of living, whereas for Cooprovaseb 
ron que a través de la cooperativa han logrado mejorar su forma de vivir, en cambio para los asociados de Cooprovaseb el móvil para mantener su nivel de vida es la venta de la producción a intermediarios, concluyendo que la cooperativa no ha influido en la mejora como personas.

Palabras clave: desarrollo, empresarial, humano, infraestructura, intermediarios, cooperativa.

$\mathrm{N}$ icaragua es el país centroamericano que cuenta con el mayor número de cooperativas registradas. El total asciende a 5678 , entre las que predominan las del sector agropecuario, con un $71 \%$. Las cooperativas generan más empleo directo que cualquier otro modelo empresarial (Flores, 2007). Las cooperativas en Nicaragua representan uno de los principales sectores de la economía del país, proveen ingresos nacionales, además de su capacidad de generar empleo para la comunidad, y su aporte a la producción del país.

El Instituto de Desarrollo Rural (IDR) promueve el desarrollo de pequeños y medianos productores en cooperativas agropecuarias y forestales, a través del fortalecimiento de capacidades de transformación productiva, asociativa empresarial y del mejoramiento de los servicios a la producción que promueve, facilitando la generación de valor agregado a sus productos (IDR, 2011).

Dentro de la estructura organizativa del IDR aparece IDR-PROCAVAL que es un proyecto que contiene componentes como inserción en cadenas de valor; diversificación de fuentes de ingresos; fortalecimiento y desarrollo de capacidades; y seguridad alimentaria (IDR, 2011). Como parte de una política económica nacional surge PROCAVAL IDR, en respuesta a la deficiente estructura productiva de Nicaragua, la que carece de infraestructura productiva, y capacidades empresariales para la innovación de procesos productivos, que mejoren el posicionamiento de los productos del país.

El proceso de desarrollo empresarial de estas organizaciones, es el principal reto del IDR/Procaval, implica que proveedores de materia prima se conviertan en empresarios rurales ofertando productos diferenciados a los que genérica y tradicionalmente han ofertado, a su vez, tener la capacidad de penetrar en mercados diferentes y mantenerse, es decir, hacer sostenible la gestión empresarial, para lo cual cuentan con el apoyo decidido del Gobierno a través del IDR (IDR, 2009).

El estudio aborda el proceso de inserción de cooperativas hortícolas de la zona seca de Matagalpa pertenecientes a PROCAVAL, partiendo de su nivel de desarrollo empresarial y tomando en consideración el enfoque del IDR, al incluir el análisis del desarrollo humano de los asociados de estas organizaciones. partners, the maintaining of their standard of living is associated to selling the produce to distributors, concluding that the cooperative did not influence the improvement of the people.

\section{DISEÑO METODOLÓGICO}

De acuerdo al objetivo y método de abordaje del problema, la investigación realizada es de tipo descriptiva, de corte transversal, con enfoque filosófico cuantitativo.

En la recolección de información se aplicó el método teórico, basado en fuentes bibliográficas, y el método empírico por medio de técnicas como la encuesta, entrevistas, grupo focal y guía de observación. Para determinar la muestra de Cooprahort, R.L se aplicó un muestreo probabilístico y en Cooprovaseb R.L un muestreo no probabilístico o intencional.

Población y muestra. La población total de socios de la cooperativa Cooprahort, R.L es de 43 socios, para la determinación de la muestra se utilizó muestreo probabilístico, tomando como criterio el pertenecer a PROCAVAL. En el caso de la cooperativa Cooprovaseb, R.L, la misma cuenta con 22 socios, se utilizó un muestreo, intencional utilizando los criterios de que fueran productores activos y que existiera accesibilidad hacia sus viviendas.

Al aplicar la fórmula del muestreo probabilístico, se determinó una muestra de 38 cooperados en la cooperativa Cooprahort, R.L. Al considerar los criterios seleccionados para la determinación de la muestra intencional en la cooperativa Cooprovaseb, R.L, se determinó que ocho socios cumplían con los mismos (productores activos con accesibilidad hacia sus viviendas).

Técnicas e instrumentos. En Cooprahort, R.L los grupos focales se realizaron en las comunidades Las Delicias de Darío y Carreta Quebrada de Sébaco. En Las Delicias asistieron 22 socios, con los que se formaron dos grupos. En Carreta Quebrada se convocaron a 21 socios, con los cuales se organizaron dos grupos. Los grupos focales se realizaron con 43 socios que es la población total de Cooprahort.

La guía de observación en Cooprahort fue realizada con los productores de ambas comunidades, y geográficamente más accesibles. Se contó con el apoyo de cinco socios de la comunidad Las Delicias. En la comunidad Carreta Quebrada también se dispuso del acompañamiento de tres socios. Este instrumento también fue aplicado en el centro de acopio y oficinas administrativas.

En Cooprahort las entrevistas estuvieron dirigidas al presidente y vicepresidente de la cooperativa, también se le aplicó a la contadora, al representante de la cooperativa ante el Programa de Inserción a Cadenas de Valor (PICV), y se recurrió a entrevistas abiertas con asociados para aclarar detalles adicionales a los establecidos en el instrumento. 
En Cooprovaseb, no se pudieron realizar los grupos focales debido a la nula asistencia; a esta cooperativa únicamente se le aplicó encuestas y guía de observación y fue necesario recurrir a entrevistas abiertas para detalles adicionales, y compensar con ello la ausencia de grupo focal.

En Cooprovaseb, R.L, la guía de observación se realizó con socios que son productores activos, se ubican geográficamente accesible, y son los únicos visitados por la técnica del IDR. Este instrumento se trabajó con cuatro socios que representan el $50 \%$ de la muestra.

Para la obtención de información sobre el funcionamiento de PROCAVAL, se aplicó entrevista al coordinador del proyecto en la zona seca, a la planificadora del IDR, y entrevistas abiertas con la persona que atiende a ambas cooperativas. Para determinar el nivel de desarrollo empresarial de cada cooperativa se utilizó el instrumento de caracterización a las organizaciones, el cual fue elaborado por el IDR. En él se establece una ponderación para cada área de análisis.

Para medir la calidad de vivienda, se utilizó el índice de calidad de vivienda propuesto por Rojas (2009). Consiste en medir siete componentes: pared, techo, piso, acceso a agua potable, energía eléctrica y tamaño de la vivienda; sin embargo, en la presente investigación se sustituyó el componente de tamaño de la vivienda por componente servicio sanitario. Ponderando cada elemento en cada uno de los componentes.

En la determinación del nivel de desarrollo humano que presenta cada cooperativa se elaboró un instrumento basado en la teoría de necesidades que plantea Maslow, en el cual se ponderó cada uno de los indicadores utilizados; agrupándolos en necesidades de déficit y de desarrollo. En la medición del desarrollo humano, se utilizó una escala, la cual indica que con un valor mayor de 85 se considera desarrollo humano alto, entre 41 y 85 , nivel de desarrollo humano medio y menor de 40, nivel de desarrollo humano bajo (Rojas, 2009).

\section{RESULTADOS Y DISCUSIÓN}

Programa cadena de valor del IDR desde las cooperativas Cooprovaseb, R.L y Cooprahort, R.L

Generalidades. En el marco de la implementación de la estrategia reforzada de crecimiento económico y reducción de la pobreza (ERCEP), el gobierno de Nicaragua ejecuta a través del Instituto de Desarrollo Rural (IDR), el programa de apoyo para la inserción de los pequeños productores en las cadenas de valor y acceso a mercado-PROCAVAL en veintisiete municipios de los departamentos de Jinotega, Matagalpa y Boaco (IDR, 2012).

Enfoque de PROCAVAL. El enfoque del proyecto responde a la necesidad de generar valor agregado de la producción primaria, la que tienen mayor peso en pequeños producto- res, con escaza capacidad de proyección empresarial en el mercado, lo que implica un abanico de clientes, así como capacidad de negociación. De igual manera Procaval se enfoca en el fortalecimiento de las capacidades empresariales de las cooperativas de las zonas rurales, con acciones orientadas a que pequeños y medianos productores puedan desarrollar la cadena de valor de los diferentes rubros de producción. Un aspecto fundamental es que dentro de la lógica del proyecto se incluye un enfoque humanista, teniendo conciencia de un desarrollo integral, que involucre al individuo.

Programación y financiamiento de PROCAVAL. Como parte de una política nacional Procaval es financiado por organismos internacionales, los que facilitan el proceso de industrialización del país. Esta circunstancia, en el corto plazo es un beneficio, sin embargo, en el largo plazo significa comprometer recursos nacionales para pago de deuda, con lo que se fomenta el círculo vicioso de la pobreza. La programación financiera de PROCAVAL es parte de la programación del Presupuesto General de la República.

Proyecto de inserción en cadenas de valor de la producción hortícola de Sébaco, Darío y Terrabona en el período 2011-2013. En el departamento de Matagalpa se realizó análisis de los rubros de mayor impacto por zonas agroecológicas; definiéndose zona seca: Sébaco, San Isidro, Darío y Terrabona con los rubros de fríjol, arroz, hortalizas y miel; zona húmeda: Rancho Grande, Waslala y La Dalia y Zona Semi húmedas: San Ramón, San Dionisio, Esquipulas, Río Blanco, y Matiguas, donde predomina los rubros de café, fríjol, cacao y leche (López, 2009).

Caracterización de los principales actores del proyecto. Los actores directos del proyecto son los pequeños productores de hortalizas, los cuales presentan problemas económicos. Para estos productores resulta difícil obtener crédito para sus actividades productivas, y comercializar las hortalizas porque son productos perecederos. Otro aspecto que limita el accionar de los productores es la tendencia al trabajo individual, lo cual dificulta la inserción a cadenas de valor. Actualmente gracias a este proyecto estos productores se unen para lograr obtener mercados justos y mejorar sus niveles de vida.

Aspectos técnicos y productivos del proyecto y descripción de la infraestructura, maquinaria y equipos. El proyecto contempla la construcción de dos centros de almacenamiento y transformación de cebolla y tomate; estos centros contaran con todo lo necesario para crear las condiciones óptimas para la generación del valor agregado, y por ende todo lo necesario para la inserción en la cadena de valor del rubro hortalizas.

En el caso de la Cooprovaseb y Cooprahort el proyecto está relativamente bien enfocado, ya que ambas cooperativas son productoras de hortalizas. 
Dentro de la empresa cooperativa de agroindustria, uno de los principales equipos para darle valor agregado a la materia prima será un deshidratador, el cual transformará estos productos con el objetivo de aumentar su vida útil, y a la vez en las épocas donde haya sobreproducción la misma sea acopiada con el fin de que sea ofertada al mercado cuando exista escases de productos y así mejorar los ingresos de los pequeños productores de las zonas aledañas.

\section{Cooperativismo}

\section{Ley General de cooperativas}

Apropiación de Ley General de Cooperativas \# 499. En el caso de Cooprovaseb un mayor número de asociados tienen dominio de la ley, en comparación con Cooprahort. Sin embargo, existe una paradoja entre ambas cooperativas, ya que Cooprahort es la que funciona mejor como cooperativa, y es donde existe mayor integración entre socios. En el caso de Cooprovaseb es una organización bajo la cual se agrupan productores que se esfuerzan de manera individual, para alcanzar sus fines. En Cooprahort 84 por ciento de los socios están apropiados de la ley general de cooperativas, mientras que en Cooprovaseb el 87 por ciento.

Órganos de gestión y toma de decisión. En Cooprahort los órganos de gestión, desde la asamblea general, se encuentran constituidos en un $100 \%$, y todos los órganos ejercen sus funciones, lo que garantiza el buen funcionamiento operativo. Otro punto importante es que existe un órgano adicional, es el comité de abastecimiento, el cual se encarga del óptimo funcionamiento del centro de acopio.

En Cooprovaseb no todos los órganos de gestión se encuentran constituidos, y además de los pocos que existen no todos sus miembros ejercen sus funciones; sólo la Asamblea General representa un $100 \%$. A las reuniones asisten generalmente 12 socios de lo que se deduce que no todos los socios están activos. A la convocatoria hecha para levantar la información no asistieron por haberse programado en la mañana (Morales, 2012).

La toma de decisión es un elemento fundamental en toda organización, ya que determina el rumbo de las operaciones administrativas, financieras y productivas; pero este elemento adquiere un carácter particular en una Cooperativa, ya que el poder de decisión recae sobre todos los socios que la integran, es decir que cualquier decisión que se tome responde a las necesidades de la mayoría de asociados.

\section{Desarrollo empresarial}

Productores activos y tipología de productores. En Cooprahort el $100 \%$ de socios que integran esta cooperativa son productores activos de hortalizas, de estos el 3\% no levantaron producción, el factor principal para ello fue que fueron afectados por plagas y enfermedades, sin embargo, ya están con planes para producir la próxima temporada. En Cooprovaseb, el $55 \%$ de socios son productores activos de hortaliza, lo que significa que el $45 \%$ de ellos son comercializadores y otros simplemente socios sin ejercer ninguna actividad primaria dentro de la cooperativa.

Los pequeños productores se consideran aquellos que tienen menos de dos manzanas de hortalizas; los medianos entre dos a cinco manzanas y los grandes más de cinco manzanas (actualmente todos los socios de las ocho organizaciones son pequeños) (IDR, 2011).

Rubro productivo. Cooprahort es una cooperativa diversificada, siendo los principales rubros de producción tomate, pepino, y chiltoma. Además de hortalizas producen granos básicos pero con un porcentaje de producción relativamente bajo.

De igual manera, Cooprovaseb es una cooperativa hortícola, aunque menos diversificada en comparación con Cooprahort. Su principal rubro de producción es pepino, seguido de cebolla y frijol. En esta cooperativa los granos básicos constituyen un rubro de producción con significativo peso económico; sin embargo, el contar con destacada producción de cebolla, justifica su incorporación en el Programa de Inserción a Cadenas (PICV).

Bienes de calidad. El bajo poder de negociación de las cooperativas de cara al mercado es una de las debilidades identificadas en el proceso de diagnósticos organizacional, esto es de particular importancia tomando en cuenta la perecibilidad de los productos ofertados, así como factores propios de su dinámica como son el manejo de volúmenes relativamente pequeños por cada cooperativa, poca especialización de las cooperativas en el comercio, poca sistematización de la información del mercado nacional de hortalizas para la planificación del negocio, entre las principales (IDR, 2011).

La calidad de bienes de cada cooperativa se analizó de acuerdo a la línea que se establece en el PICV, tomando en cuenta los requerimientos del mercado, es por eso que en este acápite se presentan los canales de comercialización de ambas cooperativas y se incluye el parámetro de Buenas Prácticas Agrícolas que es el manejo de cultivo con prácticas amigables al medio ambiente, para obtener un producto inocuo (Hernández, 2012).

Dentro del estudio de los canales de comercialización se consideraron las principales dificultades que enfrentan los socios para ubicar la producción en el mercado. En ambas cooperativas, las principales dificultades que se presentan al momento de comercializar son el bajo precio al que se comercializan las hortalizas en el mercado nacional, seguido de la plaza, es decir que éstas instituciones tienen que diversificar su abanico de clientes. Solamente el ocho por ciento no considera estas variables como dificultades.

Cadena de valor. El enfoque de cadena de valor es una herramienta que facilitó el estudio completo del rubro de hortalizas en la zona seca; identificando las limitantes que tienen 
las cooperativas tanto en la producción, transformación y comercialización de hortalizas; y también las fortalezas con la que ya cuentan estas cooperativas en cada eslabón.

Cooprovaseb no cuenta con cadena de valor; más sin embargo tiene una oportunidad con PROCAVAL/IDR. Con lo que podrá mejorar la producción (Acceso a tecnología, implementación de BPA, y BPM); Transformación (Tecnología para almacenamiento y procesamiento; así como en comercialización (uso de marca, patente, código de barra, posicionándose en mejores canales de comercialización).

Tabla 1. Cadena de valor de Cooprovaseb

\begin{tabular}{ll}
\hline Indicador & Resultado en Cooprovaseb \\
\hline Indicador & Resultado en Cooprovaseb. \\
Producción & $\begin{array}{l}\text { El nivel actual de producción no es el óptimo } \\
\text { porque no se cuenta con los recursos }\end{array}$ \\
& suficientes para cultivar (Morales, 2012). \\
& Cada productor comercializa independiente \\
Comercialización & y por eso se han afiliado al proyecto del IDR \\
& (Morales, 2012). \\
& A raíz de la falta de financiamiento hay productores \\
& que le venden a los intermediarios, los cuales \\
& financian producción para que luego los prioricen a ellos, \\
& los intermediarios tienen mayor poder de negociación \\
& (Morales, 2012).
\end{tabular}

\section{Desarrollo humano}

Ingreso. La economía campesina se caracteriza por un ingreso familiar bajo. Está formada, casi siempre, por varios productos agropecuarios, por productos no agrícolas o artesanías y por salarios obtenidos fuera de la parcela en la actividad agropecuaria o fuera de ella (Valderrama \& Mondragón, 1998).

En ambas cooperativas (Cooprahort y Cooprovaseb) las actividades agrícolas constituyen la principal, actividad para la generación de ingresos. Esta actividad genera el 100 por ciento de los ingresos de los cooperados. Un segmento de los asociados tiene negocios propios (16 y 25\% respectivamente). Seis por ciento de los ingresos de los cooperados de Cooprahort reciben remesas del exterior.

Para la gran mayoría de los cooperados de ambas cooperativas el integrarse a las cooperativas ha significado un incremento de los ingresos, 97 por ciento en COOPRAHORT y 63 por ciento en COOPROVASEB. Por otro lado, 84 por ciento de los cooperados de COOPRAHORT indica que la mejora en la satisfacción de las necesidades se debe a la integración a la cooperativa, en cambio la mayoría de los cooperados de COOPROVASEB (75\%) indica que las actividades agrícolas les han permitido mejora en la satisfacción de sus necesidades.

Con el análisis de las fuentes de ingresos se pretende establecer la dependencia que presentan los asociados de cada cooperativa, de una fuente determinada para la satisfacción de necesidades básicas, así como la diversificación de ingresos. Ambas cooperativas tienen diferentes caminos, ya sea la integración a la cooperativa, así como negocios propios. Lo anterior permite que el tema de ingreso en estas entidades sea dinámico, que no solo dependa de los ingresos por ser parte de una cooperativa.

Vivienda. Los socios de ambas cooperativas disponen de una vivienda adecuada; es decir una vivienda que cumple con los parámetros necesarios para garantizar el bienestar de las personas que habitan. La vivienda cuenta con los servicios básicos tales como energía y agua potable, así como techo, paredes, piso y servicio sanitario que proporcionan una vivienda adecuada. Los socios disponen de las condiciones necesarias para que el espacio habitable garantice el bienestar de su familia.

En ambas cooperativas el índice de calidad de vivienda (ICV), está dentro de los parámetros necesarios para que una familia cuente con una vivienda digna. COOPROVASEB tiene un índice de calidad de vivienda mayor que COOPRAHORT.

Tabla 2. Índice de calidad de vivienda

CPared CPiso CTecho CAEnergía CAAgua CSSanit ICV

\begin{tabular}{llllllll}
\hline Cooprahort & 0.66 & 0.54 & 0.66 & 0.49 & 0.98 & 0.40 & 0.622 \\
Cooprovaseb & 0.45 & 0.79 & 0.66 & 0.50 & 1.00 & 0.42 & 0.645 \\
\hline
\end{tabular}

Acceso a servicios básicos. El 100\% de asociados a Cooprahort y Cooprovaseb cuentan con agua potable, energía eléctrica y caminos. Los dos primeros componentes forman parte del índice de calidad de vivienda, en cambio, el acceso a caminos se relaciona con la rentabilidad de la actividad económica que realizan los productores, ya que facilita la movilización de la producción hacia puntos de comercialización.

En Cooprovaseb el 88\% de socios afirmó disponer de transporte. Estos socios se encuentran geográficamente más dispersos, lo que limita su fácil movilización. De igual manera la mayoría de asociados dispone del servicio de puesto de salud ( 88 y 92\% para Cooprahort y Cooprovaseb respectivamente). Cabe destacar que los socios de ambas cooperativas habitan en comunidades accesibles.

Educación y seguridad. La educación y el acceso al conocimiento, se consideran como agentes de primer orden en la multiplicación de capacidades, la generación de oportunidades, y el incremento de calidad de vida. Un desarrollo humano, bien entendido relaciona la formación de las personas con su salud, educación e ingreso (Morras, 2009).

Seguridad es la satisfacción con la alimentación de la familia, con las condiciones de la vivienda, seguridad dentro de la vivienda, seguridad de recibir financiamiento, y asistencia técnica (Cáseres, 2009). 
El análisis de seguridad se realizó tomando en consideración las condiciones que generan Cooprahort y Cooprovaseb para cada uno de sus asociados, es decir el escudo que representa la cooperativa para poder solucionar cada uno de los conflictos a los que se enfrentan los socios. Los temores que cada socio tiene son los elementos que socavan su propia seguridad y el impacto que estos tengan afecta directamente la calidad de vida, y es aquí donde la cooperativa desempeña un papel importante como móvil de desarrollo.

\section{CONCLUSIONES}

El Programa de Cadena de Valor del IDR consiste en la construcción de cuartos fríos para alargar la vida útil de las hortalizas, y el uso de un horno deshidratador de cebolla.

El funcionamiento que tiene Cooprahort y Cooprovaseb como cooperativas es completamente opuesto. Cooprahort funciona bajo los lineamientos que se establecen en la Ley General de Cooperativas \# 499, caso contrario Cooprovaseb, que es una organización con nula coordinación.

Cooprahort tiene alta competitividad empresarial, ya que cuenta con una eficiente gestión organizacional; es una cooperativa con rostro humano que contribuye al desarrollo de la comunidad. En competitividad Cooprovaseb es el polo opuesto a Cooprahort, no cuenta con capital de trabajo como cooperativa, ni infraestructura productiva por lo que tampoco tiene la capacidad de ser responsable socialmente con las comunidades aledañas.

El desarrollo humano de los socios de Cooprahort y Cooprovaseb, es similar, en ingreso, vivienda y servicios básicos; aunque existen notables diferencias en la participación de socios dentro de la cooperativa.

Según criterios del IDR Cooprahort tiene un nivel de desarrollo empresarial medio, en cambio el nivel de desarrollo empresarial de Cooprovaseb es deficiente. En el nivel de desarrollo humano Cooprahort tiene un nivel alto, y Cooprovaseb presenta nivel medio.

\section{REFERENCIAS BIBLIOGRÁFICAS}

Cáseres, SV. (2009). Impacto de PRODECOOP R.L, en el desarrollo humano de asociados (as) a sus cooperativas de base en la Región I, Las Segovias, Nicaragua. Puebla, México: Colegio de Postgraduados.

Flores, J. 2007. Una Mirada al movimiento cooperativo. Nuevo Diario, Managua, Ni, Sep. 10:15 B.

Hernández, G. (30 de Noviembre de 2012). Rotación de Cultivo. (W. C. López, \& J. M. Palma, Entrevistadores)

IDR. 2012. Instituto de Desarrollo Rural. Recuperado el 29 de marzo de 2012, de Instituto de Desarrollo Rural: http://www.idr.gob ni/idr_institucional.html

IDR (Instituto de Desarrollo Rural). 2011. Manual Operativo IDR-PROCAVAL. Matagalpa, Nicaragua: IDR.

IDR. (15 de Enero de 2012). Orígenes y Desarrollo del Instituto de Desarrollo Rural. Recuperado el 20 de Abril de 2012, de Orígenes y Desarrollo del Instituto de Desarrollo Rural: http://www.idr.gob.ni-IDR-2012.html

IDR. (2011). PICV HORTALIZAS. Matagalpa, Nicaragua: IDR.

IDR. (24 de febrero de 2012). Programa de Apoyo a Pequeños Productores para la Inserción a las Cadenas Productivas. Recuperado el 15 de Abril de 2012, de Programa de Apoyo a Pequeños Productores para la Inserción a las Cadenas Productivas: http://www. idr.gob.ni/Programas_IDR_2012.html

IDR. (2012). Programa del IDR. Matagalpa, Nicaragua: IDR.

IDR. (2009). Propuesta de Estrategia de Desarrollo Empresarial IDR/PROCAVAL. Matagalpa, Nicaragua: IDR.

López, E. (2009). Mapeo y Focalización de Actores en las Cadenas de Hortalizas y Miel mediante la recopilación de Información Secundaria en 15 municipios en el Departamento de Matagalpa. Matagalpa, Nicaragua: IDR, Delegación Matagalpa.

Morales, M. (24 de Agosto de 2012). Cooperativismo, Desarrollo Empresarial, Desarrollo Humano. (W. C. López, \& J. M. Palma, Entrevistadores)

Morras, E. (2009). fao.org. Obtenido de fao.org: ftp://ftp.fao.org/TC/TCA/ESP/pdf/nica/bloqueII_2.pdf

Rojas, J. (2009). Cooperativismo y Desarrollo Humano: Una propuesta metodológica para su medición. Matagalpa: Apante.

Valderrama, M., \& Mondragón, H. (1998). Desarrollo y equidad con campesinos. Santa Fe, Colombia: IICA en coedición con TM Editores. 